\title{
MR-basierte CT-ähnliche Bildgebung und simulierte konventionelle Radiographie
}

Die konventionelle Röntgendiagnostik stellt bei Knochentumoren nach wie vor den wesentlichen Bestandteil der Bildgebung dar. Lokalisation, Destruktionsmuster und Periostreaktionen geben Aufschluss über die biologische Aktivität von Knochenläsionen [1]. Röntgenmorphologie und Matrixmineralisationsmuster erlauben oftmals eine spezifische Diagnose oder erhebliche Einengung der Differenzialdiagnose. Die Röntgendiagnostik stellt somit die Weichen für das weitere Vorgehen [2, 3]. Die Computertomographie (CT) hilft zusätzlich bei der Evaluation von Knochenläsionen in anatomischen Regionen, in denen die Radiographie, bedingt durch Überlagerung, nur eingeschränkt beurteilbar ist $[4,5]$. Aufgrund der mit der Röntgen- und CTDiagnostik verbundenen Strahlenbelastung wird insbesondere für die Anwendung bei jungen Patienten mit benignen Knochenläsionen, wiederholten Untersuchungen bzw. radiologischen Verlaufskontrollen nach Verfahren gesucht, die ohne den Einsatz ionisierender Strahlung eine vergleichbare diagnostische Aussagekraft liefern können.

Nordeck und Mitarbeiter konnten in einer radiologischen Studie zeigen, dass die Simulation röntgenähnlicher Bilder anhand von 3D-MRT-Datensätzen möglich ist [6]. Diese Bilder basierten auf Protonendichte-gewichteten MRT-Sequenzen. Ziel der Studie war es, mit einer kontrastreichen Darstellung der Kortikalis Winkelmessungen am Sprunggelenk zu ermöglichen [6]. Die Studie zeigte diesbezüglich eine hohe Übereinstimmung zwischen konventionellen und simulierten Röntgenbildern [6]. Bei der Bildgebung von Knochentumoren ist jedoch nicht nur die Darstellung der Kompakta, also letztlich der Außenkontur des Knochens, sondern auch die Visualisierung des trabekulären Knochens von entscheidender Bedeutung. Hierfür eignen sich insbesondere T1-gewichtete Gradienten-Echo-Sequenzen, die die Grundlage für einen Algorithmus zur Simulation von Röntgenbildern bieten können [4]. Wir

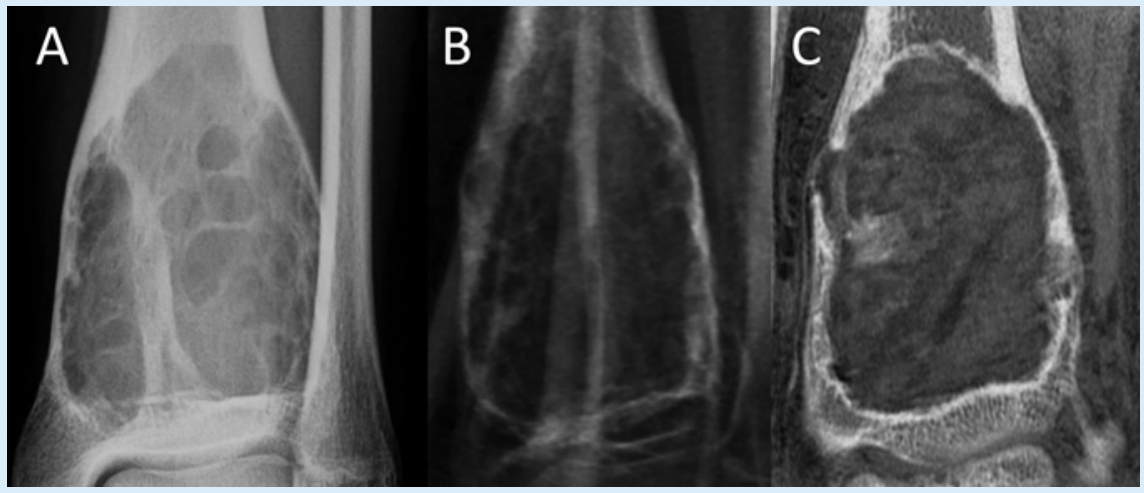

• Abb. 1 Histologisch gesichertes intraossäres Angiosarkom in der distalen Tibia einer 18-jährigen Patientin. (A) Die konventionelle Röntgenaufnahme zeigt eine inhomogene lytische Läsion mit endostaler Ausdünnung der Kortikalis. Die gleiche Morphologie wird mittels (B) MRT-basiertem und simuliertem Röntgenbild sowie (C) MR-basiertem, CT-ähnlichen Bild visualisiert.

haben diese Methode bei Patienten mit Knochentumoren angewandt, bei denen zuvor im Rahmen der klinischen Diagnostik konventionelle Röntgenbilder akquiriert wurden [4]. Die aus den MRT-Datensätzen berechneten simulierten Röntgenbilder und CTähnlichen Bilder wurden mit konventionellen Röntgenbildern verglichen. Die Sensitivität und Spezifität MRT-basierter Datensätze bei der Klassifikation der Knochenläsionen in aggressive und nichtaggressive Läsionen waren vergleichbar mit der Sensitivität und Spezifität konventioneller Röntgenbilder, wobei die histologische Diagnose als Referenzstandard diente ( $\triangleright$ Abb. 1 ). Als Vorteil der MRT-basierten, CT-ähnlichen Bilder und simulierten Röntgenbilder gegenüber konventionellen Röntgenbildern zeigte sich, dass zusätzliche Informationen über extraossäre Tumorkomponenten und die Tumorarchitektur generiert werden konnten.

\section{Zusammenfassung}

Basierend auf einer hochaufgelösten MRTSequenz, können CT-ähnliche Bilder sowie simulierte Röntgenbilder generiert werden, anhand derer Knochentumoren mit einer der konventionellen Diagnostik vergleichbaren Sicherheit klassifiziert werden können.
Zusätzlich können aus den synthetischen Bildern wesentliche Informationen zur Tumorarchitektur und -ausdehnung gewonnen werden. Der Einsatz dieser MRT-Sequenz in der muskuloskelettalen Diagnostik könnte in Zukunft einen Beitrag zur Reduktion der Strahlenexposition von Patienten führen.

\section{Autorinnen / Autoren}

Priv.-Doz. Dr. med. Alexandra S. Gersing Institut für diagnostische und interventionelle Radiologie, Klinikum rechts der Isar, Technische Universität München

Prof. Dr. med. Klaus Woertler

Institut für diagnostische und interventionelle Radiologie, Klinikum rechts der Isar, Technische Universität München

\section{Korrespondenzadresse}

Priv.-Doz. Dr. med. Alexandra S. Gersing Institut für diagnostische und interventionelle Radiologie

Klinikum rechts der Isar

Technische Universität München Ismaningerstraße 22

81675 München - Deutschland

Tel. +498941406351

Fax +49 8941404994

E-Mail: alexandra.gersing@tum.de 
[3] Sweet DE, Madewell JE, Ragsdale BD. Radiologic and pathologic analysis of solitary bone lesions. Part III: Matrix patterns. Radiol Clin North Am 1981; 19: 785-814

[4] Gersing AS, Gersing AS, Pfeiffer D, Kopp FK, Schwaiger B], Knebel C, Haller B, Noël PB, Settles M, Rummeny EJ, Woertler K. Evaluation of MR-derived CT-like images and simulated radiographs compared to conventional radiography in patients with benign and malignant bone tumors. Eur
Radiol 2018. doi:10.1007/s00330-0185450-y:

[5] Miller TT. Bone tumors and tumorlike conditions: Analysis with conventional radiography. Radiology 2008; 246: 662-674

[6] Nordeck SM, Koerper CE, Adler A, Malhotra V, Xi Y, Liu GT, Chhabra A. Simulated radiographic bone and joint modeling from 3D ankle MRI: Feasibility and comparison with radiographs and 2D MRI. Skeletal Radiol 2017; 46: 651664 